\title{
Paediatric case series of drug reaction with eosinophilia and systemic symptoms (DRESS): 12-year experience at a single referral centre in Hong Kong and the first reported use of infliximab
}

Department of Paediatrics and Adolescent Medicine, Queen Mary Hospital, Hong Kong

\section{KeYwORDS}

paediatric; drug reaction with eosinophilia and systemic symptoms; DRESS; drug-induced hypersensitivity syndrome; Chinese; Hong Kong

\section{Corresponding author}

Gilbert T Chua

Room 115, New Clinical Building

Queen Mary Hospital

102 Pokfulam Road, Hong Kong

Phone: 0085222554482

E-mail: gtc510@gmail.com

\section{Doi}

10.23822/EurAnnACI.1764-1489.47

\begin{abstract}
Summary
DRESS (drug reaction with eosinophilia and systemic symptoms) is a rare but potentially life-threatening disorder characterized by fever, skin eruption, haematological abnormalities and multi-organ dysfunction after drug exposure. The pathophysiology is thought to be related to interactions between culprit drugs, viral reactivation and T-lymphocytes activation. We report 4 paediatric patients with DRESS who were treated at our centre over the past 12 years. Most cases improved after corticosteroids. Other immunosuppressive medications were attempted in refractory cases with varied outcomes. Patient 3 was the first reported case that involved the use of infliximab, a TNF- $\alpha$ inhibitor, for DRESS. Although clinical efficacy was not observed for this one patient, a previous study demonstrated that patients with DRESS, disease progression and HHV-6 reactivation had elevated pre-treatment TNF- $\alpha$ and IL-6 levels. Further research is needed to explore the role of these cytokines in DRESS..
\end{abstract}

\section{Introduction}

Drug reaction with eosinophilia and systemic symptoms (DRESS), also known as drug-induced hypersensitivity syndrome, is a rare but potentially life-threatening disorder characterized by fever, skin eruption, haematological abnormalities and multi-organ involvement. There are three proposed diagnostic criteria (table I). The estimated incidence ranges from 1 in 1,000-10,000 drug exposures, with a mortality rate of $10 \%$. The typical clinical course involves a latency period of 3 to 76 days after culprit drug exposure $(1,2)$. Here we present 4 Chinese paediatric patients with DRESS managed at our centre over the past 12 years who experienced highly variable clinical courses. Most of these cases of DRESS im- proved after the administration of high-dose corticosteroids, while in refractory cases other immunosuppressive medications were attempted with variable outcomes.

\section{Case Summaries}

\section{Patient 1}

A 13-year-old boy with chronic urticaria and asthma first presented in May 2001, with autoimmune hepatitis suspected to be an idiosyncratic drug reaction related to doxepin and famotidine. This diagnosis was made based on neutrophilic and eosinophilic liver infiltration evident in a liver biopsy, and disease resolution after pulse methylprednisolone 
and high dose prednisolone were given. Two months later, he presented again with fever, cough and urticarial exacerbation while prednisolone was weaned. Amoxicillin-clavulanate was prescribed empirically for 10 days, but his neutrophilia and eosinophilia persisted. The prednisolone dose was increased for suspected flare of autoimmune hepatitis.

Nevertheless, he developed progressive respiratory distress requiring ventilatory support. Meropenem and clarithromycin were empirically administered. Sepsis workup including blood culture and bronchoalveolar lavage was negative. High resolution computed tomography (HRCT) and lung biopsy confirmed autoimmune pneumonitis and bronchiolitis obliterans with organizing pneumonia. He also developed multiple skin ulcers, and a skin biopsy confirmed a drug eruption. The overall features were compatible with DRESS triggered by amoxicillin-clavulanate. Subsequently, four doses of weekly pulse methylprednisolone and an eight-week course of cyclophosphamide were given, followed by oral prednisolone for 2 years and azathioprine for 3 years. His skin condition, lung function and repeat HRCT showed gradual disease resolution. He remained in clinical remission with no further exacerbations for more than 15 years of follow up.

\section{Patient 2}

A 5-year-old girl with a complex congenital heart disease status-post surgical repair complicated by subsequent left-sided stroke and focal seizures was started on carbamazepine after confirming negative HLA-B1502 status. Two months later, in February 2014, she developed generalized and blistering erythroderma, conjunctivitis, oral ulcers and fever. Blood testing demonstrated eosinophilia (1.3 x 10\%/L), 21\% atypical lymphocytes and deranged liver function. Serum cytomegalovirus pp65 antigen and oral ulcer swab for herpes simplex virus (HSV) were positive, and therefore ganciclovir was given. Human herpesvirus type-6 (HHV-6) and HHV7 DNA PCR were negative. Stevens-Johnson syndrome was initially suspected, so carbamazepine was discontinued while intravenous immunoglobulin (IVIG) and prednisolone were administered. Her rash improved and her skin biopsy confirmed a drug eruption.

Despite these treatments, she suffered from progressive liver dysfunction, and a liver biopsy revealed vanishing bile duct syndrome. She also began to have renal impairment, acute pancreatitis and pneumonitis. The overall presentation was compatible with DRESS.

Table I - Diagnostic criteria for drug reaction with eosinophilia and systemic symptoms (DRESS).

\begin{tabular}{|c|c|c|}
\hline Bocquet, Bagot and Roujeau Criteria & SCAR-J & RegiSCAR \\
\hline $\begin{array}{l}\text { Drug rash } \\
\text { Hematological abnormalities: } \\
\text { - eosinophilia }>1.500 / \mathrm{mm}^{3} \\
\text { - presence of atypical lymphocytes. } \\
\text { Systemic involvement: } \\
\text { - adenopathy > } 2 \mathrm{~cm} \text { in diameter } \\
\text { - hepatitis (increase in transaminases at } \\
\text { least twice of normal values) } \\
\text { - interstitial nephritis } \\
\text { - pneumonitis } \\
\text { - carditis. }\end{array}$ & $\begin{array}{l}\text { Paculopapular rash developing more } \\
\text { than } 3 \text { weeks after starting therapy } \\
\text { with a limited number of drugs. } \\
\text { Persistent clinical findings after drug } \\
\text { withdrawal. } \\
\text { Fever }>38^{\circ} \mathrm{C} \text {. } \\
\text { Hepatic abnormalities } \\
\text { (alt }>100 \mathrm{u} / \mathrm{l}) \text {. } \\
\text { Leucocyte abnormalities with } \\
\text { the presence of at least one of the } \\
\text { following: } \\
\text { - leucocytosis }>11.000 / \mathrm{mm}^{3} \\
\text { - atypical lymphocytosis }>5 \% \\
\text { - eosinophilia }>1.500 / \mathrm{mm}^{3} \text {. } \\
\text { HHV-6 reactivation. }\end{array}$ & $\begin{array}{l}\text { Hospitalization. } \\
\text { Reaction suspected to be drug related. } \\
\text { Acute skin rash. } \\
\text { Fever above } 38^{\circ} \mathrm{C} \text {. } \\
\text { Enlarged lymph nodes at } \geq 2 \text { sites. } \\
\text { Involvement of at least one internal organ. } \\
\text { Blood count abnormalities: } \\
\text { - lymphocytes above or below the normal } \\
\text { range } \\
\text { - eosinophils above the normal range (in } \\
\text { percentage or absolute count) } \\
\text { - platelets below the normal range. }\end{array}$ \\
\hline $\begin{array}{l}\text { All } 3 \text { criteria required, with at least } 1 \\
\text { hematologic and } 1 \text { systemic feature } \\
\text { included. }\end{array}$ & $\begin{array}{l}\text { Typical DRESS syndrome: presence } \\
\text { of } 7 \text { findings; } \\
\text { Atypical DRESS syndrome when the } \\
\text { first } 5 \text { findings are present. }\end{array}$ & $\begin{array}{l}\text { Patients with the first } 3 \text { findings and } 3 \text { out } \\
\text { of } 4 \text { systemic features will enter a scoring } \\
\text { system ranging from }-4 \text { to } 9 \text { points to decide } \\
\text { whether the case is definite, probable or } \\
\text { possible for DRESS. }\end{array}$ \\
\hline
\end{tabular}


Her clinical course remained stormy, complicated by corticosteroid-induced duodenal ulcer resulting in massive bleeding requiring endoscopic haemostasis. Despite aggressive antimicrobial therapies and supportive measures, she died of disseminated infections.

\section{Patient 3}

A previously healthy 14-year-old girl presented with Salmonella paratyphi A septicaemia in June 2007. She was treated with a week of ceftriaxone and co-trimoxazole upon discharge. One week later, she developed fever, a generalized maculopapular and blistering rash, bilateral conjunctivitis and hepatosplenomegaly (figure 1). Blood testing demonstrated eosinophilia $(1.18 \times 10 \%$ L) and $26 \%$ atypical lymphocytes, cholestatic liver derangement and coagulopathy. Ultrasound of the liver was suggestive of cholangitis, and her skin biopsy confirmed a drug eruption.

The patient was diagnosed with DRESS due to co-trimoxazole and she was started on prednisolone. Her liver dysfunction and coagulopathy improved, but her skin condition did not. A course of pulse methylprednisolone was given, followed by prednisolone, azathioprine, cyclosporine A and mycophenolate mofetil (MMF). Monthly IVIG, infliximab, topical corticosteroids, acitretin and phototherapy with narrow band UVB and PUVA were attempted but these therapies did not result in any improvement. Her skin condition was further complicated by photosensitivity after ultraviolet therapy, which led to erythroderma and skin exfoliation. Therefore, phototherapy was withheld. Her skin disease

Figure 1 - Patient 3, who presented with erythroderma and a blistering skin eruption.

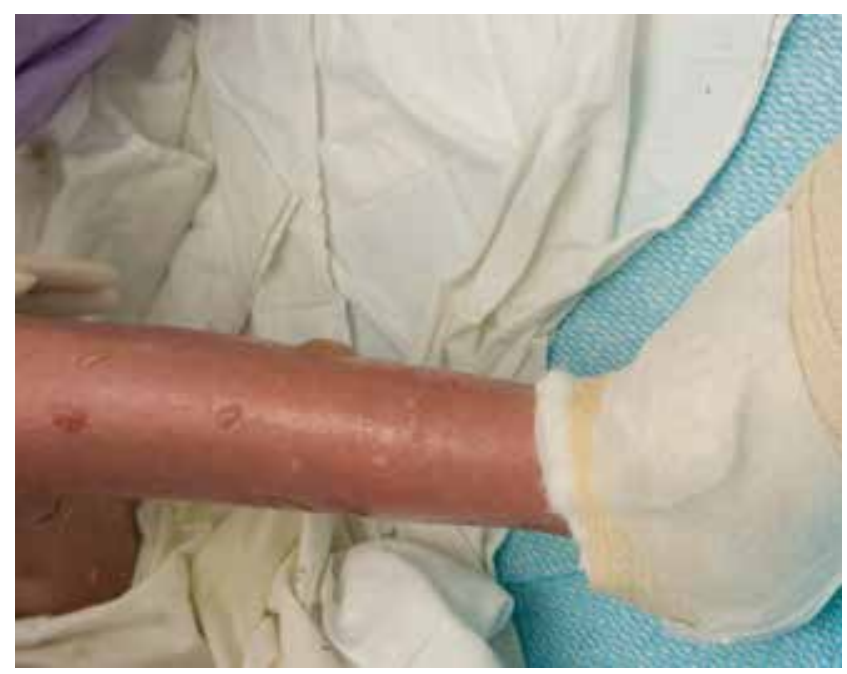

eventually evolved into psoriatiform lesions and waxy papulosis with significant palmoplantar keratoderma. Weekly methotrexate was given for four years which was able to stabilize her skin condition.

Five years after her DRESS diagnosis, she developed Graves' disease requiring carbimazole and later radioactive iodine ablation. Her latest dermatological assessment showed she had generalized vitiligo and alopecia totalis.

\section{Patient 4}

This is a 17-year-old female with juvenile idiopathic arthritis and $\mathrm{IgG}_{2}$ deficiency, who initially presented in August 2007 with tonsillitis treated with co-trimoxazole. Nine days later, she developed an erythematous, maculopapular rash over her face and body, massive lymphadenopathy, fever, acute renal failure and respiratory failure. Microbiological investigations were unrevealing. Her respiratory and skin condition deteriorated, and she required extracorporeal membrane oxygenation support (ECMO). Skin biopsy was suggestive of a drug reaction and the overall picture was compatible with DRESS triggered by co-trimoxazole.

Her condition was also complicated by pseudomembranous colitis and acute cholangitis. Immunological investigations showed persistent hypogammaglobulinemia and B-cell lymphopenia. The patient was managed with corticosteroids and her skin condition gradually improved. She was weaned off from ECMO and continued to receive prednisolone and monthly IVIG for her chronic hypogammaglobulinemia.

\section{Discussion}

The four Chinese paediatric DRESS patients described have variable disease courses and outcomes. While using the proposed diagnostic criteria was helpful in making the diagnosis, identification of the culprit drug remained challenging. Drug patch test may be used to identify the culprit drug, which is commonly utilized to diagnose non-IgE mediated cutaneous adverse drug reactions or delayed drug hypersensitivity. It is recommended to be performed at least six months after the disappearance of adverse drug reactions. Positive predictive value of the test varies between different drugs. The sensitivity and specificity appears to be higher for certain anticonvulsants, such as carbamazepine, and antimicrobials, such as beta-lactams, but lower for medications such as allopurinol and salazopyrin (3). However, skin patch tests were unable to be performed in our cases since testing supplies were not available for patients 1 and 4 at the time they presented, while patient 2 was critically ill and the skin condition for patient 3 was not suitable for the test all along. Therefore, identification of the culprit drugs in these 
cases mainly relied on their clinical history and the temporal sequences of the events.

The mainstay management approach to DRESS includes avoidance of unnecessary empirical use of medications during the acute phase of disease to minimize potential immune cross-reactivity, early recognition and withdrawal of the culprit drug, and aggressive immunosuppressive therapies and supportive measures (4). The first-line treatment remains to be high-dose corticosteroids, which are generally effective during the acute phase. For long-term treatment, or in steroid-unresponsive cases, steroid-sparing agents may be used. Cyclosporine (5-7), IVIG (8-11), and cyclophosphamide (12) have been reported to be effective in treating steroid-refractory DRESS. However, there has been no randomized trial so far comparing the efficacies between these agents.

As demonstrated in our cases, patients with DRESS have highly variable clinical courses and responses to immunosuppressive agents. To our knowledge, patient 3 was the first reported case of DRESS that involved the use of infliximab, a TNF- $\alpha$ inhibitor. Although clinical efficacy was not observed for this patient, large-scale studies using infliximab and other immunomodulating therapies are required to fully determine the optimal treatment for patients with DRESS refractory to corticosteroids. Moreover, Uno et al. demonstrated that elevated pre-treatment levels of TNF- $\alpha$ and IL- 6 in patients with DRESS and HHV-6 reactivation were correlated with disease progression, and therefore TNF- $\alpha$ and IL-6 levels may potentially serve as a biomarker for this syndrome (13). Further research is needed to explore the role of TNF- $\alpha$ and IL- 6 in DRESS.

\section{Acknowledgement}

We thank doctors TL Lee, CK Yeung, NC Fong, SY Chan, CY Chan and the nursing staff for their dedicated clinical care of these patients.

\section{Conflict of interest}

The authors declare that they have no conflict of interest.

\section{References}

1. Husain Z, Reddy BY, Schwartz RA. DRESS syndrome: Part I. Clinical perspectives. J Am Acad Dermatol 2013; 68(5):693.e1-14.

2. Criado PR, Avancini J, Santi CG, et al Drug reaction with eosinophilia and systemic symptoms (DRESS): a complex interaction of drugs, viruses and the immune system. Isr Med Assoc J 2012; 14(9):577-582.

3. Barbaud A. Skin testing and patch testing in non-IgE-mediated drug allergy. Curr Allergy Asthma Rep 2014; 14(6):442.

4. Husain Z, Reddy BY, Schwartz RA. DRESS syndrome: Part II. Management and therapeutics. J Am Acad Dermatol 2013; 68(5):709.e1-9; quiz 718-20. doi: 10.1016/j.jaad.2013.01.032. Review.

5. Kirchhof MG, Wong A, Dutz JP. Cyclosporine Treatment of Drug-Induced Hypersensitivity Syndrome. JAMA Dermatol 2016; 152(11):1254-1257.

6. Zhang ZX, Yang BQ, Yang Q.Treatment of drug-induced hypersensitivity syndrome with cyclosporine. Indian J Dermatol Venereol Leprol 2017; 83(6):713-717.

7. Zuliani E, Zwahlen H, Gilliet F, et al. Vancomycin-induced hypersensitivity reaction with acute renal failure: resolution following cyclosporine treatment. Clin Nephrol 2005; 64:155-158.

8. Scheuerman O, Nofech-Moses Y, Rachmel A, et al. Successful treatment of antiepilepticdrug hypersensitivity syndrome with intravenous immune globulin. Pediatrics 2001; 107:e14.

9. Fields KS, Petersen MJ, Chiao E, Tristani-Firouzi P. Case reports: treatment of nevirapine-associated dress syndrome with intravenous immune globulin (IVIG). J Drugs Dermatol 2005; 4:510513.

10. Darban M, Bagheri B. Drug Reaction with Eosinophilia and Systemic Symptoms Induced by Valproic Acid: A Case Report. Iran Red Crescent Med J 2016; 18(9):e36825.

11. Galvão VR, Aun MV, Kalil J. Clinical and laboratory improvement after intravenous immunoglobulin in drug reaction with eosinophilia and systemic symptoms. J Allergy Clin Immunol Pract 2014; 2(1):107-110.

12. Laban E, Hainaut-Wierzbicka E, Pourreau F, et al. Cyclophosphamide therapy for corticoresistant drug reaction with eosinophilia and systemic symptoms (DRESS) syndrome in a patient with severe kidney and eye involvement and Epstein-Barr virus reactivation. Am J Kidney Dis 2010; 55:e11-4.

13. Uno H, Kabashima K, Tohyama M, et al. TNF-a as a useful predictor of human herpesvirus- 6 reactivation and indicator of the disease process in drug-induced hypersensitivity syndrome (DIHS)/ drug reaction with eosinophilia and systemic symptoms (DRESS). J Dermatol Sci 2014; 74(2):177-179. 